\title{
CONFIDENTIEL
}

NOM DE L'ÉTUDE:

PRODUIT À L'ÉTUDE:

FORME GALENIQUE/DOSAGE:

TITRE DE L'ÉTUDE:

INVESTIGATEUR Coordinateur:

PROMOTEUR:

CHEF DE PROJET:

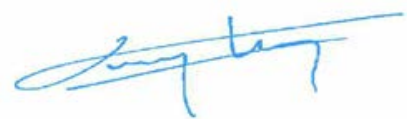

Signature du Chef de Projet
AMBIDEX

AmBisome $^{\circledR}$ (amphotéricine B liposomale)

$10 \mathrm{mg} / \mathrm{kg}$

AmBiDex - Traitement préemptif des colonisations multiples à candida chez des patients de réanimation présentant un sepsis.

Pr Elie AZOULAY

Service de Réanimation médicale

Hôpital Saint Louis

1 avenue Claude Vellefaux

75010 PARIS

France

Tél. : 0142499421

FOVEA

3 Bis Chemin de la Jonchère

92500 Rueil Malmaison

France

Tel. : 0147140495

Dr Jean François DREYFUS

\section{JUIN 2008}

\section{Date}

PROPRIETE EXCLUSIVE DU GROUPE FOVEA NE DOIT ETRE COMMUNIQUE, HORS DU GROUPE, QU'AUX PERSONNES QUI, DE PAR LEUR FONCTION ET/OU LEUR ROLE DANS L'ETUDE, DOIVENT AVOIR ACCES AU CONTENU INTEGRAL DU PROTOCOLE.

J'ai lu ce protocole et ses annexes et j'accepte de conduire l'étude selon ses dispositions. 


\section{AMENDEMENT AU PROTOCOLE N²}

\section{JUSTIFICATION DE L'AMENDEMENT}

Cet amendement $n^{\circ} 2$ au protocole a 4 objectifs :

- Prolonger la période de recrutement, en raison du nombre insuffisant de patients inclus à ce jour,

- Modifier le critère d'inclusion concernant la créatinémie, en vue de permettre l'inclusion de patients ayant une créatinémie comprise entre 220 et $300 \mu \mathrm{mol} / \mathrm{L}$, ceux-ci étant très représentés dans la population cible de l'étude. L'inclusion de ces patients sera laissée à l'appréciation de l'investigateur, eu égard au rapport bénéfice/risque pour le patient.

- Modifier le critère d'exclusion concernant les traitements antifongiques antérieurs reçus par le patient, et n'exclure que les patients ayant reçu de l'Ambisome ${ }^{\circledR}$ depuis leur admission en service de réanimation

- Préciser le délai d'obtention du nombre de sites colonisés

- Ajouter, aux visites 1, 4, 5 et 6 le dosage du béta-D-glucane, marqueur prédictif des infections fongiques

\section{SECTIONS Calendrier de l'étude ET Strucuture administrative de l'étude :}

Début de l'étude : $\quad$ Septembre 2007

Fin de l'étude : $\quad$ Mars 2008

Modifié par:

Début de l'étude : $\quad$ Septembre 2007

Fin prévisionnelle de l'étude : Avril 2009 (dernier patient dernière visite)

\section{SECTION 1.5 Problématique de l'étude}

Une étude américaine récente place les infections à candida spp. au quatrième rang des infections nosocomiales les plus rencontrées avec un taux de mortalité de $40 \%$. De plus, les infections à candida spp. surviennent majoritairement dans les services de réanimation $(48,49)$. La colonisation à candida n'est pas liée à un accroissement de mortalité mais le continuum colonisation - infection à candida, démontré en 1994 par Pittet et ses collègues souligne l'importance de la prévention d'une invasion chez les patients à haut risque de candidose invasive (16). De plus, le retour d'une hémoculture positive à candida signant une candidémie fréquemment mortelle est un événement tardif et peu sensible.

Ces faits sont en faveur d'un traitement antifongique précoce qui permettrait de traiter efficacement une candidose systémique à une étape où les techniques de diagnostic n'ont pas la sensibilité nécessaire pour affirmer ou infirmer l'infection. Ce traitement serait mené chez des patients hospitalisés en réanimation avec une colonisation par candida dans au moins deux sites et à haut risque de candidose invasive. 


\section{Modifié par :}

Une étude américaine récente place les infections à candida spp. au quatrième rang des infections nosocomiales les plus rencontrées avec un taux de mortalité de $40 \%$. De plus, les infections à candida spp. surviennent majoritairement dans les services de réanimation $(48,49)$. La colonisation à candida n'est pas liée à un accroissement de mortalité mais le continuum colonisation - infection à candida, démontré en 1994 par Pittet et ses collègues souligne l'importance de la prévention d'une invasion chez les patients à haut risque de candidose invasive (16). De plus, le retour d'une hémoculture positive à candida signant une candidémie fréquemment mortelle est un événement tardif et peu sensible.

Ces faits sont en faveur d'un traitement antifongique précoce qui permettrait de traiter efficacement une candidose systémique à une étape où les techniques de diagnostic n'ont pas la sensibilité nécessaire pour affirmer ou infirmer l'infection. Ce traitement serait mené chez des patients hospitalisés en réanimation avec une colonisation par candida dans au moins deux sites (au minimum un site extra digestif colonisé au moment de l'inclusion du patient, le deuxième pouvant être localisé dans un délai de 96 heures suivant son inclusion) et à haut risque de candidose invasive.

Par ailleurs, le Béta-D-glucane sera utilisé pour étayer le diagnostic de l'infection fongique invasive (50) chez les patients inclus dans cette étude.

\section{SECTION 1.6 Objectifs de l'étude :}

\section{Objectifs secondaires}

Les objectifs secondaires consistent à évaluer les paramètres suivants :

- Morbidité selon trois critères :

Durée d'hospitalisation

Durée de séjour en réanimation

Nombre de jours sans défaillance organique

- Incidence d'infection fongique invasive selon les critères de l'EORTC-MSG modifiés

- Survie à 28 jours

\section{Modifié par :}

\section{Objectifs secondaires}

Les objectifs secondaires consistent à évaluer les paramètres suivants :

- Morbidité selon trois critères :

Durée d'hospitalisation

Durée de séjour en réanimation

Nombre de jours sans défaillance organique

- Incidence des infections fongiques invasives selon les critères de l'EORTC-MSG modifiés, et prenant en compte l'utilisation du beta-D-glucane comme critère supplémentaire du diagnostic des infections fongiques invasives

- Survie à 28 jours 


\section{SECTION 3.1 Critères d'inclusion :}

Age $\geq 18$ ans

Ventilation mécanique depuis plus de $48 \mathrm{~h}$

Patients présentant une défaillance d'organe en plus de celle requérant la ventilation mécanique

Colonisation à candida d'au moins 2 sites dont 1 non digestif

Suspicion de sepsis nosocomial

Mise en place d'une nouvelle antibiothérapie pour la suspicion de sepsis nosocomial

Consentement de la personne de confiance au moment de l'inclusion

\section{Modifié par :}

Age $\geq 18$ ans

Ventilation mécanique depuis plus de $48 \mathrm{~h}$

Patients présentant une défaillance d'organe en plus de celle requérant la ventilation mécanique

Colonisation à candida d'au moins 2 sites dont un non digestif : après documentation d'un premier site extra-digestif (poumon, urines, muqueuses, redons ...), la mise en évidence d'un deuxième site devra obligatoirement intervenir dans un délai de 96 heures

Suspicion de sepsis nosocomial

Mise en place d'une nouvelle antibiothérapie pour la suspicion de sepsis nosocomial

Consentement de la personne de confiance au moment de l'inclusion

\section{SECTION 3.2 Critères de non inclusion :}

Traitement par antifongiques par voie orale (décontamination digestive sélective) ou par voie systémique dans les 15 jours précédant l'inclusion

Patients répondant aux critères de mise en œuvre d'un traitement antifongique classique

Patients moribonds (SAPS à l'inclusion >65)

Patients neutropéniques ou receveurs d'une greffe de cellules souches hématopoïétiques ou d'organe solide ou bénéficiant d'une chimiothérapie

Présence d'une infection fongique documentée (prouvée ou probable) selon les critères de l'EORTC/MSG modifiés

Créatininémie $>220 \mu \mathrm{mol} / \mathrm{L}$ ou hémodialyse

Grossesse

\section{Modifié par :}

Traitement par Ambisome ${ }^{\circledR}$ depuis l'admission dans le service de réanimation pour l'épisode de sepsis actuel

Patients répondant aux critères de mise en œuvre d'un traitement antifongique classique

Patients moribonds (SAPS à l'inclusion >65)

Patients neutropéniques ou receveurs d'une greffe de cellules souches hématopoïétiques ou d'organe solide ou bénéficiant d'une chimiothérapie

Présence d'une infection fongique documentée (prouvée ou probable) selon les critères de l'EORTC/MSG modifiés

Hémodialyse ou Créatininémie $>\mathbf{3 0 0} \mu \mathrm{mol} / \mathrm{L}$, avec pour les valeurs de Créatininémie comprises entre 220 et $300 \mu \mathrm{mol} / \mathrm{L}$ une inclusion laissée au jugement de l'investigateur eu égard au rapport bénéfice/risque

Grossesse 


\section{SECTION 5.8 Recueil des informations tout au long de l'étude :}

\begin{tabular}{|c|c|c|c|c|c|c|c|}
\hline & V1 (J1) & V2 (J2) & V3 (J3) & V4 (J8) & V5 (J14) & V6 (J21) & $\begin{array}{l}\text { V7 (J28 ou } \\
\text { Fin d'essai) }\end{array}$ \\
\hline $\begin{array}{l}\text { Signature du consentement éclairé avant } \\
\text { toute procédure liée à l'étude }\end{array}$ & $x$ & & & & & & \\
\hline Démographie & $\mathrm{X}$ & & & & & & \\
\hline Critères d'inclusion et de non inclusion & $\mathrm{X}$ & & & & & & \\
\hline Comorbidités à l'inclusion & $X$ & & & & & & \\
\hline Signes vitaux & $x$ & $x$ & $\mathrm{X}$ & $x$ & $x$ & $\mathrm{X}$ & $\mathrm{X}$ \\
\hline Score SOFA & $x$ & $x$ & $\mathrm{X}$ & $\mathrm{X}$ & $\mathrm{X}$ & $X$ & $\mathrm{X}$ \\
\hline Critères de sepsis & $\mathrm{X}$ & $X$ & $\mathrm{X}$ & $x$ & $\mathrm{X}$ & $\mathrm{X}$ & $\mathrm{X}$ \\
\hline Laboratoire & $x$ & $x$ & $x$ & $\mathrm{X}$ & $\mathrm{X}$ & $X$ & $\mathrm{X}$ \\
\hline Colonisation à candida & $X$ & \multicolumn{6}{|c|}{$2 \mathrm{X} /$ semaine } \\
\hline Survenue d'infection fongique & & $x$ & $x$ & $x$ & $\mathrm{X}$ & $\mathrm{X}$ & $\mathrm{X}$ \\
\hline Administration du produit à l'étude & $X$ & & & $X$ & & & \\
\hline Traitements concomitants & $x$ & $\mathrm{X}$ & $\mathrm{X}$ & $X$ & $\mathrm{X}$ & $X$ & $\mathrm{X}$ \\
\hline Evénements indésirables & $x$ & $\mathrm{X}$ & $\mathrm{X}$ & $X$ & $\mathrm{X}$ & $\mathrm{X}$ & $\mathrm{X}$ \\
\hline Recueil survie & $X$ & $X$ & $X$ & $X$ & $X$ & $X$ & X \\
\hline
\end{tabular}




\section{Modifié par :}

Une partie des échantillons de sang prélevés lors des visites V1, V4, V5 et V6 (7 ml à chaque visite) sera conservée jusqu'à la fin de l'étude (dernière visite du dernier patient) dans le centre investigateur, puis envoyé au laboratoire de l'Hôpital St Louis, Avenue Claude-Vellefaux - 75010 Paris, en vue du dosage du Béta-D-Glucane. Ces prélèvements sanguins ne seront pas utilisés à des fins d'analyse génétiques.

\begin{tabular}{|c|c|c|c|c|c|c|c|}
\hline & V1 (J1) & V2 (J2) & V3 (J3) & V4 (J8) & V5 (J14) & V6 (J21) & $\begin{array}{l}\text { V7 (J28 ou } \\
\text { Fin d'essai) }\end{array}$ \\
\hline $\begin{array}{l}\text { Signature du consentement éclairé avant } \\
\text { toute procédure liée à l'étude }\end{array}$ & $x$ & & & & & & \\
\hline Démographie & $\mathrm{x}$ & & & & & & \\
\hline Critères d'inclusion et de non inclusion & $\mathrm{X}$ & & & & & & \\
\hline Comorbidités à l'inclusion & $x$ & & & & & & \\
\hline Signes vitaux & $\mathrm{x}$ & $\mathrm{x}$ & $\mathrm{x}$ & $\mathrm{x}$ & $\mathrm{x}$ & $\mathrm{x}$ & $\mathrm{x}$ \\
\hline Score SOFA & $\mathrm{X}$ & $\mathrm{x}$ & $\mathrm{X}$ & $\mathrm{X}$ & $\mathrm{X}$ & $\mathrm{x}$ & $\mathrm{X}$ \\
\hline Critères de sepsis & $x$ & $x$ & $x$ & $\mathrm{x}$ & $x$ & $\mathrm{x}$ & $\mathrm{x}$ \\
\hline Laboratoire & $x$ & $x$ & $x$ & $x$ & $x$ & $\mathrm{x}$ & $x$ \\
\hline Colonisation à candida & $\mathrm{x}$ & \multicolumn{6}{|c|}{$2 \mathrm{X} /$ semaine } \\
\hline Survenue d'infection fongique & & $\mathrm{X}$ & $\mathrm{x}$ & $\mathrm{X}$ & $\mathrm{X}$ & $\mathrm{X}$ & $\mathrm{X}$ \\
\hline Administration du produit à l'étude & $x$ & & & $x$ & & & \\
\hline Traitements concomitants & $\mathrm{X}$ & $\mathrm{X}$ & $\mathrm{X}$ & $\mathrm{x}$ & $\mathrm{x}$ & $\mathrm{x}$ & $\mathrm{x}$ \\
\hline Evénements indésirables & $\mathrm{X}$ & $\mathrm{X}$ & $\mathrm{x}$ & $\mathrm{X}$ & $\mathrm{X}$ & $\mathrm{X}$ & $\mathrm{X}$ \\
\hline Recueil survie & $x$ & $x$ & $x$ & $x$ & $x$ & $\mathrm{x}$ & $x$ \\
\hline Dosage du Beta-D-Glucane & $\mathrm{X}$ & & & $\mathrm{X}$ & $\mathrm{x}$ & $\mathrm{x}$ & \\
\hline
\end{tabular}




\section{SECTION 6.3.2 Critères secondaires d'évaluation :}

Les critères secondaires d'évaluation de l'efficacité comprennent :

- L'évaluation des événements indésirables non liés au traitement, survenus durant les 28 jours de l'étude.

- L'évaluation des paramètres de morbidité suivants :

- $\quad$ Durée de séjour dans l'unité de Soins Intensifs

- $\quad$ Durée de séjour à l'hôpital

- $\quad$ Nombre de jours sans défaillance d'organe

- L'incidence des infections fongiques invasives selon les critères de l'EORTC-MSG

- L'incidence d'autres infections nosocomiales subséquentes

- Le taux de mortalité à 28 jours

Modifié par :

Les critères secondaires d'évaluation comprennent :

- L'évaluation des événements indésirables non liés au traitement, survenus durant les 28 jours de l'étude.

- L'évaluation des paramètres de morbidité suivants :

- $\quad$ Durée de séjour dans l'unité de Soins Intensifs

- $\quad$ Durée de séjour à l'hôpital

- $\quad$ Nombre de jours sans défaillance d'organe

- L'incidence des infections fongiques invasives selon les critères de l'EORTC-MSG, et prenant en compte l'utilisation du beta-D-glucane comme critère supplémentaire du diagnostic des infections fongiques invasives

- L'incidence d'autres infections nosocomiales subséquentes

- Le taux de mortalité à 28 jours

\section{SECTION 9 Références bibliographiques :}

\section{Ajouté :}

50. Z. Odabasi, G. Mattiuzzi, E. Estey, H. Kantarjian, F. Saeki, R. J. Ridge, P. A. Ketchum, M.A. Finkelman, J. H. Rex and L. Ostrosky-Zeichner

Beta-d-Glucan as a Diagnostic Adjunct for Invasive Fungal Infections : Validation, Cutoff Development, and Performance in Patients with Acute Myelogenous Leukemia and Myelodysplastic Syndrome 


\section{Synopsis de l'étude :}

Modifié en consequence par :

\begin{tabular}{|c|c|}
\hline Titre de l'étude & $\begin{array}{l}\text { AmBiDex - Traitement préemptif des colonisations multiples à candida chez des } \\
\text { patients de réanimation présentant un sepsis }\end{array}$ \\
\hline Justification & $\begin{array}{l}\text { La candidémie est associée à une mortalité élevée or, il n'existe pas de procédure } \\
\text { diagnostique qui permette d'anticiper la survenue de cette pathologie, mais il a été } \\
\text { démontré, chez les patients chirurgicaux en soins intensifs, qu'il existait un } \\
\text { continuum qui va de la colonisation à l'infection par candida. Il est donc urgent de } \\
\text { développer des stratégies thérapeutiques lorsqu'un patient présente des } \\
\text { colonisations de plusieurs sites qui coexistent avec des facteurs de risque comme } \\
\text { la défaillance de plusieurs organes (y compris la nécessité d'une ventilation } \\
\text { assistée) et un état septique nosocomial sévère. } \\
\text { La quasi-totalité des patients qui ont présenté une candidémie présentaient une } \\
\text { colonisation par candida. et il semble que la colonisation soit associée à une } \\
\text { prolongation de la durée du séjour en soins intensifs. } \\
\text { L'hypothèse de base de cette étude est que les patients en Unité de Soins Intensifs } \\
\text { présentant une colonisation de plusieurs sites ont un risque élevé de candidose } \\
\text { invasive et que l'administration précoce d'un agent antifongique empêchera } \\
\text { l'apparition d'une candidose invasive. } \\
\text { Une administration hebdomadaire d'AmBisome pourrait représenter une option } \\
\text { thérapeutique importante que l'on peut justifier sur la base des résultats } \\
\text { pharmacocinétiques et du profil pharmacodynamique, car elle associe un schéma } \\
\text { d'administration simple à un large spectre d'activité thérapeutique. }\end{array}$ \\
\hline $\begin{array}{l}\text { Nombre de } \\
\text { centres prévus }\end{array}$ & 9 centres en France \\
\hline Objectif & $\begin{array}{l}\text { Principal: } \\
\begin{array}{l}\text { Tolérance et sécurité d'emploi de l'AmBisome }{ }^{\circledR} \quad 10 \mathrm{mg} / \mathrm{kg} \text { en une } \\
\text { administration hebdomadaire (2 perfusions à une semaine d'intervalle) }\end{array} \\
\text { Secondaires: } \\
\text { - Évaluation des paramètres de morbidité suivants: } \\
\text { Durée de séjour dans l'Unité de Soins Intensifs } \\
\text { Durée de séjour à l'hôpital } \\
\text { Nombre de jours sans défaillance d'organe } \\
\text { - Incidence des infections fongiques invasives selon les critères de l'EORTC-MSG } \\
\text { modifiés, et prenant en compte l'utilisation du beta-D-glucane comme } \\
\text { critère supplémentaire du diagnostic des infections fongiques invasives } \\
\text { - Incidence d'autres infections nosocomiales subséquentes } \\
\text { - Taux de mortalité à } 28 \text { jours }\end{array}$ \\
\hline
\end{tabular}




\begin{tabular}{|c|c|}
\hline Plan expérimental & Étude pilote, multicentrique, réalisée en ouvert \\
\hline $\begin{array}{l}\text { Nombre } \\
\text { patients }\end{array}$ & 30 \\
\hline Population cible & $\begin{array}{l}\text { Patients en unité de soins intensifs présentant une colonisation de plusieurs sites } \\
\text { par candida dans le contexte d'une défaillance d'au moins deux organes (y compris } \\
\text { la nécessité d'une assistance respiratoire) et d'un état septique nosocomial sévère }\end{array}$ \\
\hline Traitement & AmBisome $^{\circledR} 10 \mathrm{mg} / \mathrm{kg}$ (amphotéricine B liposomale) \\
\hline $\begin{array}{l}\text { Durée } \\
\text { traitement }\end{array}$ & 2 perfusions à une semaine d'intervalle \\
\hline $\begin{array}{l}\text { Diagnostic et } \\
\text { principaux critères } \\
\text { d'éligibilité }\end{array}$ & $\begin{array}{l}\text { 1. Age } \geq 18 \text { ans } \\
\text { 2. Ventilation depuis plus de } 48 \text { heures } \\
\text { 3. Patients présentant une défaillance d'organe en plus de la ventilation } \\
\text { mécanique } \\
\text { 4. Colonisation à candida d'au moins } 2 \text { sites dont un non digestif : après } \\
\text { documentation d'un premier site extra-digestif (poumon, urines, } \\
\text { muqueuses, redons ...), la mise en évidence d'un deuxième site devra } \\
\text { obligatoirement intervenir dans un délai de } 96 \text { heures } \\
\text { 5. Suspicion de sepsis nosocomial } \\
\text { 6. Mise en place d'une nouvelle antibiothérapie pour la suspicion de sepsis } \\
\text { nosocomial } \\
\text { 7. Consentement de la personne de confiance au moment de l'inclusion }\end{array}$ \\
\hline $\begin{array}{l}\text { Critères de } \\
\text { non-inclusion }\end{array}$ & $\begin{array}{l}\text { 1. Traitement par Ambisome }{ }^{\circledR} \text { depuis l'admission dans le service de } \\
\text { réanimation pour l'épisode de sepsis actuel } \\
\text { 2. Patients répondant aux critères de mise en œuvre d'un traitement } \\
\text { antifongique classique } \\
\text { 3. Patients moribonds (SAPS à l'inclusion }>65 \text { ) } \\
\text { 4. Patients neutropéniques ou receveurs d'une greffe de cellules souches } \\
\text { hématopoïétiques ou d'organe solide ou bénéficiant d'une chimiothérapie } \\
\text { 5. Présence d'une infection fongique documentée (prouvée ou probable) selon } \\
\text { les critères de l'EORTC/MSG modifiés } \\
\text { 6. Hémodialyse ou Créatininémie }>300 \mu \text { mol/L, avec pour les valeurs de } \\
\text { Créatininémie comprises entre } 220 \text { et } 300 \mu \text { mol/L une inclusion laissée } \\
\text { au jugement de l'investigateur eu égard au rapport bénéfice/risque } \\
\text { 7. Grossesse }\end{array}$ \\
\hline
\end{tabular}


Informations recueillies et procédures

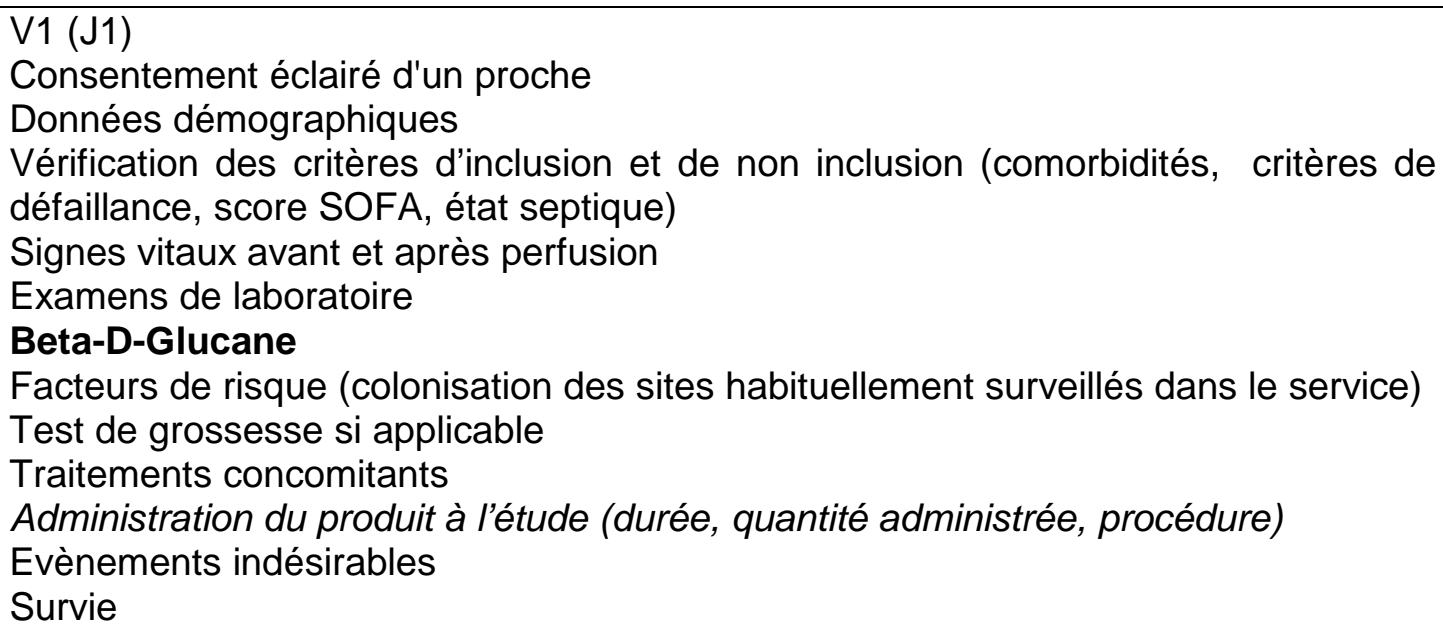

Recueil des informations sur la colonisation fongique des sites testés à V1, 2 fois par semaine

V2 (J2)

Signes vitaux, score SOFA, critères d'un sepsis, examens de laboratoire

Survenue d'infection fongique invasive, survie

Traitements concomitants, évènements indésirables

V3 (J3)

Signes vitaux, score SOFA, critères d'un sepsis, examens de laboratoire

Survenue d'infection fongique invasive, survie

Traitements concomitants, évènements indésirables

V4 (J8)

Signes vitaux, score SOFA, critères d'un sepsis, examens de laboratoire et Beta-DGlucane

Survenue d'infection fongique invasive, survie

Traitements concomitants, évènements indésirables

Administration du produit à l'étude

Événements indésirables au cours et au décours de la perfusion

V5 (J14)

Signes vitaux, score SOFA, critères d'un sepsis, examens de laboratoire et Beta-D-

Glucane

Survenue d'infection fongique invasive, survie

Traitements concomitants, évènements indésirables

V6 (J21)

Signes vitaux, score SOFA, critères d'un sepsis, examens de laboratoire et Beta-DGlucane

Survenue d'infection fongique invasive, survie

Traitements concomitants, évènements indésirables

V7 (J28 ou J SORTIE) VISITE FINALE

Signes vitaux, score SOFA, critères d'un sepsis, examens de laboratoire

Survenue d'infection fongique invasive, survie

Traitements concomitants, évènements indésirables 